Thorax (1975), 30, 516.

\title{
Primary sarcoma of the lung
}

\author{
E. W . J . C A M E R O N
}

Thoracic Surgical Unit, The Royal Infirmary, Edinburgh

\begin{abstract}
Cameron, E. W. J. (1975). Thorax, 30, 516-520. Primary sarcoma of the lung. Primary sarcoma of lung is defined to exclude sarcomata of lymphatic origin. A series of nine patients is presented, all of whom were seen in Edinburgh. In each case there is histological proof of the diagnosis of sarcoma. From the same population and during the same period of time, 6000 cases of bronchial carcinoma presented. Eight of the nine patients underwent resection of the tumour, and the pathological findings and clinical results are described. From a review of the literature and from the Edinburgh series, a general series of 59 cases of primary sarcoma of the lung has been selected. The criteria for selection were directed at the assessment of the results of surgery in the treatment of this lesion. The five-year survival rate after 'curative' surgery is $52 \%$, and the prognosis after surgery does not appear to depend on the radical nature of the operation.
\end{abstract}

Primary sarcoma of lung arises from bronchial or pulmonary parenchyma and is quite distinct in tissue of origin from those other primary sarcomata which, although found within the lung, are not of pulmonary origin. The malignant lymphoma of lung, a tumour of lymphatic tissue, is therefore excluded from consideration not only because of its histogenesis, but also because its behaviour differs markedly from that of other sarcomata. In particular, lymphatic metastases are common with lymphatic sarcoma and rare or late with other forms of sarcoma; also lymphatic tumours are highly radiosensitive (Paterson, 1963), and their management differs from that of the radioresistant pulmonary sarcoma. This distinction between primary pulmonary sarcoma and sarcoma of lymphoid origin situated in the lung is supported in the papers of Beck and Reganis (1951) and Baron and Whitehouse (1961).

The material for this paper is drawn from two sources: from a series of nine patients seen in Edinburgh over a period of 18 years, and from a review of the literature.

\section{CLINICAL MATERIAL AND PATHOLOGICAL FINDINGS}

Spencer (1968) distinguishes two groups of primary pulmonary sarcoma, those arising from bronchi or central tumours, and those arising from pulmonary tissue or peripheral tumours. However, in the Edinburgh series the exact anatomical origin is not stated in the records, and it would seem reasonable to describe sarcoma according to its cell type without attempting further subclassification.

In six of the nine Edinburgh cases the tumouro was a fibrosarcoma. Stout (1948) has described $\stackrel{\mathbb{Q}}{\circ}$ the pathological features of fibrosarcoma. It is a $\overrightarrow{\vec{\phi}}$ malignant tumour of fibroblasts which produce 3 collagen and reticulin. Stout distinguishes a differentiated form of fibrosarcoma with few mitoses. from a poorly differentiated form in which the cells are pleomorphic, with a high mitotic rate ando with immature development of collagen. $\mathrm{He}$ con $\cong$ tends that neurofibrosarcoma is not a separate entity but is more correctly termed fibrosarcoma Black (1950) has commented on the extreme rarityô of distant metastasis from primary fibrosarcoma of bronchus.

PATIENT 1 A 67-year-old woman presented with multiple complaints including rheumatoid arthritis Parkinsonism, and urinary incontinence. She had hay a malignant melanoma excised from the left arm when she was 64 years old. She had no symptoms referablew to the lungs but on chest radiography an opacity was seen in the left lower lobe. At bronchoscopy smooth, pale mass was seen in the posterior basaf bronchus. Biopsy showed this to be a fibrosarcomas The patient died while in the ward and at necropsy a $10 \mathrm{~cm}$ tumour was found lying enclosed in a fals capsule. There was no evidence of metastasis.

PATIENT 2 A 51-year-old man presented with a six month history of exertional dyspnoea and a four-week history of aching right chest pain. He had a palpable 
right scalene lymph node which was removed and was shown to have sarcoid. Radiographically there was a right upper pulmonary lesion, which at thoracotomy proved to be a tumour invading the chest wall. Right upper lobectomy was performed with resection of the second to fourth ribs. Two months later a rounded opacity was seen at the right apex and the patient developed Horner's syndrome. Radiotherapy effected no improvement and the patient died three months after operation.

PATIENT 3 A 52-year-old man was found in a mass radiography survey to have a right lower pulmonary opacity. He was asymptomatic. A $5 \mathrm{~cm}$ mass lying apparently in the oblique fissure was removed by local excision. This patient had no evidence of metastasis or recurrence of tumour nine years after operation. He died two years later; the cause of death is unknown. The resected tumour was a fibrosarcoma of lung of the differentiated type as described by Stout (1948).

PATIENT 4 An 18-year-old boy presented with a twoweek history of cough and haemoptysis. When 2 years old he had had a course of radiotherapy for a malignant thymoma; this diagnosis had been made on radiographic grounds alone. He had since been well. A chest film showed a rounded $5 \mathrm{~cm}$ opacity in the apical segment of the right lower lobe. A segmental resection was performed in the belief that the lesion was a benign tumour, a diagnosis that examination of a frozen section taken at the time of operation was unable to refute. Subsequently the patient was clinically well but in the follow-up chest film a new opacity was recognized in the right hilum, and 10 months after the first operation a $4 \mathrm{~cm}$ hilar recurrence was removed at right pneumonectomy. The patient was well six months later.

PATIENT 5 A 66-year-old woman presented with right chest pain of 10 months' duration. A right lower lobar opacity was seen on the chest film. At right thoracotomy a $10 \mathrm{~cm}$ tumour was shelled out from the surrounding compressed lung tissue. The operative diagnosis was fibroma and the pathological diagnosis well differentiated fibrosarcoma. Eighteen months after operation the patient was well.

PATIENT 6 A 34-year-old woman was found to have a right pulmonary opacity in the chest film made as part of a pre-employment medical examination. Three years previously a haemangiosarcoma in the groin had been treated by excision and postoperative radiotherapy. By right pneumonectomy the lung containing a $12 \mathrm{~cm}$ cyst was resected. At each pole of the cyst was a cap of fibrosarcoma compressing lung parenchyma. The pathological diagnosis was fibrosarcoma arising in a hamartomatous cyst. The patient was well three years six months after surgery.
Each fibrosarcoma was soft, fleshy, and rounded, was clearly demarcated from surrounding pulmonary tissue, and could be enucleated with ease. Patients 1, 2, 4, and 6 had a poorly differentiated form of tumour on histological examination.

Two of the Edinburgh series are leiomyosarcomata. It is stated by Montgomery (1965) that these tumours generally arise in a pre-existing leiomyoma. It is uncertain whether this is true of primary pulmonary leiomyosarcoma but it emphasizes the necessity of excluding a uterine primary before the tumour is regarded as primary in the lung. Both the Edinburgh patients were women.

PATIENT 7 A 50-year-old woman presented with intractable diarrhoea. A left pulmonary opacity was found on routine chest radiography. Fifteen years previously she had had a hysterectomy for fibroids. There was no evidence of malignancy on review of these sections. Eight years previously she had undergone partial gastrectomy for benign gastric ulcer. Extensive investigation of her diarrhoea revealed no relationship between it and the pulmonary lesion. A $6 \mathrm{~cm}$ mass was removed at left upper lobectomy. The patient was well 5 years later.

PATIENT 8 A 30-year-old woman presented with a six-month history of right pleuritic pain, cough and weight loss, and with a three-week history of haemoptysis. Chest radiography showed a right upper lobar opacity and the nature of the tumour was identified from biopsy material taken at bronchoscopy. At thoracotomy a $6 \mathrm{~cm}$ mass was found to involve the upper and intermediate bronchi; a pneumonectomy was performed. The patient was lost to follow-up.

Both leiomyosarcomata were rounded and firm, with a whorled appearance on the cut surface. The tumour in patient 7 could be enucleated.

Rhabdomyosarcoma is an extremely rare tumour. Only four examples are accepted by Spencer (1968) as being primary pulmonary rhabdomyosarcoma, and one of these cases (patient 9) has previously been published (Drennan and McCormack, 1960). The neoplasm is recognized by the cross-striations present in the muscular elements. Rhabdomyosarcoma is regarded as a highly malignant tumour and its most frequent site of occurrence is in the genito-urinary tract of children under 5 years of age. The theoretical explanation for occurrence of such a tumour in the lung is speculative. Womack and Graham (1938) suggested that aberrant development of 
the embryological lung bud could provide any pattern of cell derived from mesoderm. The other possibility is that during the malignant change cells may differentiate into forms not normally seen in the tissue where the tumour is growing.

PATIENT 9 A 56-year-old man presented with a sixmonth history of cough, haemoptysis, and pleuritic pain in the left chest. A left lower lobar opacity was seen in the chest radiograph, and undifferentiated tumour cells were found in the sputum. Left pneumonectomy was performed and, during manipulation of the $12 \mathrm{~cm}$ rounded mass, the false capsule burst and jelly-like material escaped. The hilar lymph nodes were resected and these were subsequently shown to contain tumour. On histological examination crossstriations were seen in the myofibrils but despite this differentiated feature the pathologist considered the tumour to be highly malignant, as evidenced by the pleomorphism of the cells, the numerous mitoses, and the invasion of lymph nodes. Four months after operation the patient died from pulmonary oedema. There was no clinical evidence of recurrence or metastasis at the time of death.

Other forms of sarcoma have not been seen in Edinburgh and are very rare in the literature. Hering et al. (1962) reported a primary pulmonary liposarcoma. The diagnosis of myxosarcoma is equivocal since myxomatous change occurs in fibrosarcomata and the distinction between the two becomes difficult. The diagnosis of primary chondrosarcoma of lung is confused by the problem of excluding an extrapulmonary primary (Lowell and Tuhy, 1949). There is no reason to believe that these tumours behave in a different fashion from other sarcomata.

\section{CLINICAL FINDINGS AND RESULTS}

The average age of patients in the Edinburgh series was 52 years with a range from 18 to 67 years, and of the nine patients four were male and five female.

Four patients $(1,3,6,7)$ at presentation had no signs or symptoms referable to the lungs.

One patient (patient 1) died before operation. Two patients ( 2 and 9$)$ died after operation with an average survival of four months. Patient 2 was known to have had recurrence of tumour.

Four patients $(3,5,6,7)$ were well and without evidence of recurrence or metastasis of tumour a mean five years after operation. Patient 4 developed recurrence of tumour and was well six months after re-operation. Patient 8 was lost to follow-up. Three patients $(3,4,5)$ underwent local excision of tumour or segmental resection, two patients ( 2 and 7 ) underwent lobectomy, and? three patients $(6,8,9)$ underwent pneumonectomy. $\frac{\bar{\sigma}}{\bar{s}}$

On examination of the resected specimens at the time of operation characteristic findings were the ease with which the tumours could bes enucleated from a false capsule and the difficulty in making an accurate clinical diagnosis. Inpatient 4 the diagnosis remained obscure despitew frozen-section examination of the tumour. Of the nine patients three had previously been treated for tumour of other organs: in patient 1 , malig $\omega$ nant melanoma of the arm; in patient 6, haemanir giosarcoma of groin; and in patient 7 , fibromar of uterus. Review of the histology of these? tumours and comparison with the pulmonary sarcomata which the patients subsequently developed ruled out the possibility that the lung tumours were secondary. Patient 4 had previouslyo undergone a course of radiotherapy for malignan $\mathbb{E}$ thymoma although this diagnosis had not been proven histologically.

\section{A REVIEW OF THE LITERATURE}

The general series referred to consists of 59 cases of primary sarcoma of lung. This series is drawni from several sources: the bibliography of caseso collected by Dyson and Trentalance (1964), the papers of Rothe (1965), Crane and Sutton (1972), Schamaun and Kunz (1973), and Becker and Donhöffner (1974), and the Edinburgh series. The criteria used in selecting cases from the above material for this general series are several, anc $\overrightarrow{5}$ the criteria are directed at the examination of the results of surgery in the treatment of pulmonary sarcoma.

Verification of the histopathology of the tumours is crucial. Dyson and Trentalance (1964) demanded good photomicrographs and a good detailed description of the tumour before its acceptance in their series, and the same basis of selection is used in the present series. By reason of adequate history and complete gross ands histopathological examination each case is thought to be a true pulmonary primary. No example of chondrosarcoma has been included because of the difficulty in determining whether the lesion is primary in the lung or not. In all cases the tumour was confined to the lung so that the tissue of origin is not in doubt, and at operation the clear:ance of the tumour was reckoned to be complete ${ }^{-}$ Any evidence of extrapulmonary spread at theD time of resection, for example to the chest walle or to the hilar lymph nodes as in patients 2 and 9 in the Edinburgh series, has meant exclusion from the general series. Every case has beero 
treated by surgery and surgery alone, and all have survived surgery and its immediate complications.

Out of a total of 94 cases of primary sarcoma of lung reviewed, 59 fitted the criteria described above. The 35 others either did not come to surgery or some other reason for exclusion existed. The longest survival after diagnosis in this group of 35 was 10 months.

Of the 59 cases in the general series, $14 \mathrm{had}$ either local excision of the tumour or wedge or segmental resection, 18 had lobectomy, and 27 pneumonectomy. The results of these operations must be interpreted in the light of a number of factors, including the histological type of the tumour, the size of the tumour, and the age of the patient.

With regard to tumour cell type, 22 were fibrosarcomata (one case each of neurofibrosarcoma and myxofibrosarcoma is included under this head), 22 were leiomyosarcomata, and the remaining 15 were described as sarcoma, no further differentiation of the histopathology being attempted. The best results of surgery are achieved with leiomyosarcoma, next fibrosarcoma, and the worst results with sarcoma. These results are summarized in the Table. However, the numbers involved and the differences between the results are too small to be of any statistical significance, and it seems reasonable to ignore tumour type in assessing the results of surgery.

The average size of tumour removed by local excision or segmental resection was $4.7 \mathrm{~cm}$ in its maximum diameter; at lobectomy the size was $5.5 \mathrm{~cm}$ and at pneumonectomy $6.3 \mathrm{~cm}$.

Age at the time of operation is not significantly different for any one form of operation.

Of 14 patients treated by local excision or segmental resection, 11 were alive with an average survival time of five years two months. The meaning of survival is that the patient was well and had no evidence of recurrence or metastasis. Three treated by local excision were dead, two from recurrence and one from unrelated cause.
These three lived for a mean of six years after operation. This type of operation was performed on four cases of leiomyosarcoma and therefore these figures are not biased by a preponderance of tumours which may, because of their histology, have a better prognosis.

Eighteen patients underwent lobectomy, nine survived for an average of four years four months, and nine died with an average survival after operation of two years nine months. Six of these deaths are known to have been due to recurrence or metastasis.

Pneumonectomy was performed in 27 cases; 12 survived for a mean of five years four months, and 15 died after living for an average period of one year 11 months after operation. Recurrence or metastasis was the recognized cause of death in six of the patients who died.

\section{DISCUSSION}

Primary sarcoma of lung is a rare tumour. Mallory (1936) found one case in 8000 consecutive necropsies, and Noehren and McKee (1954) one in 7272. The diagnosis of primary pulmonary sarcoma is by histology alone. Reference has been made to the difficulty of making the diagnosis at operation, even when frozen-section facilities are available. This view is supported by Hovorka (1966).

The highest incidence of sarcoma is during the fourth decade whereas for bronchial carcinoma the maximum incidence is during the sixth and seventh decades (le Roux, 1968). In the Edinburgh series there have been nine histologically proven cases of primary sarcoma of lung. This represents the experience of a unit covering a population of 1500000 for 18 years. Of these nine cases, eight were resected. During the same period and from the same population 6000 cases of bronchial carcinoma were seen, the resection rate for these being $37 \%$. At presentation none of the patients with sarcoma had clinical, radiographic or endoscopic evidence of metastasis of

T A B L E

SURVIVAL AFTER SURGERY ACCORDING TO TYPE OF TUMOUR

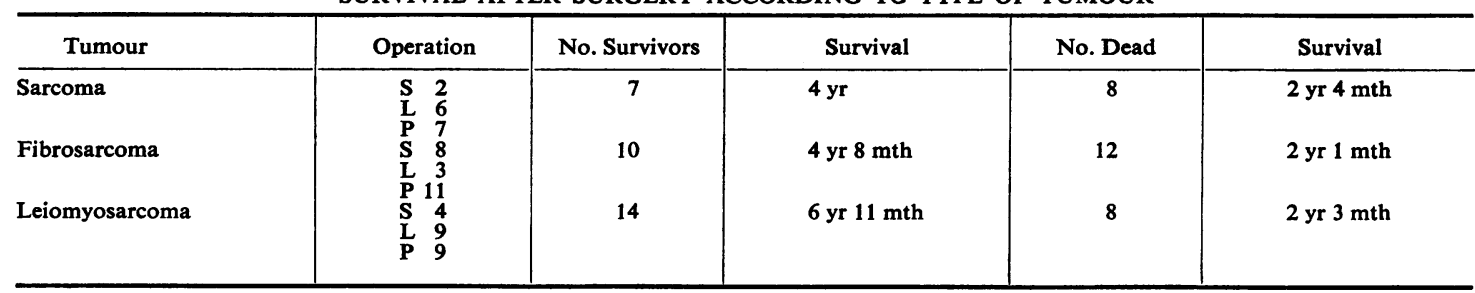

$\mathbf{S}=$ local excision or segmental resection $\mathbf{L}=$ lobectomy $; \mathbf{P}=$ pneumonectomy. 
the tumour, whereas such evidence was present in $45.9 \%$ of patients with carcinoma (le Roux, 1968).

Of the 59 cases in the general series, 31 were living free from recognized recurrence or metastasis at an average of five years five months after operation. Twenty-eight had died, the mean survival being two years three months, and of those who died 14 are known to have had recurrence of tumour and in five cases the cause of death was known to be unrelated to tumour.

A comparison can be made between the results of surgery for primary pulmonary sarcoma and the results for secondary pulmonary sarcoma and bronchial carcinoma. The five-year survival after 'curative' surgery for primary sarcoma of lung is $52 \%$, for secondary sarcoma of lung $33.8 \%$ (Feldman and Kyriakos, 1972), and for bronchial carcinoma the five-year survival after pneumonectomy is $20 \%$ and after lobectomy $38 \%$ (le Roux, 1968). There is therefore good evidence that the prognosis after surgery in cases of primary sarcoma is better than for bronchial carcinoma.

Despite the necessity for wide excision of primary sarcomata occurring in soft tissues elsewhere in the body (Bowden, 1964), in the lung local excision or segmental resection appear, at least from the evidence presented in this paper, to be as effective as more radical procedures.

I am grateful to Mr. J. D. Wade, Mr. R. J. McCormack, and Mr. P. Walbaum for permission to study the records and to publish details of patients who have been under their care.

\section{REFERENCES}

Baron, M. G. and Whitehouse, W. M. (1961). Primary lymphosarcoma of the lung. American Journal of Roentgenology, 85, 294.

Beck, W. C. and Reganis, J. C. (1951). Primary lymphoma of the lung. Journal of Thoracic Surgery, 22, 323.

Becker, H. and Donhöffner, A. (1974). Pulmonary sarcoma, clinical findings, therapy and results. Thoraxchirurgie, 22, 10.

Black, H. (1950). Fibrosarcoma of the bronchus. Journal of Thoracic Surgery, 19, 123.

Bowden, L. (1964). Clinical management of soft part sarcomas. Annals of the New York Academy of Sciences, 114, 1047.
Crane, M. and Sutton, J. P. (1972). Primary sarcoma of the lung. Southern Medical Journal, 65, 850.

Drennan, J. M. and McCormack, R. J. (1960). $\frac{\bar{\omega}}{7}$ Primary rhabdomyosarcoma of lung. Journal of $\mathbb{\AA}$ Pathology and Bacteriology, 79, 147.

Dyson, B. C. and Trentalance, A. E. (1964). Resection of primary pulmonary sarcoma. Journal of $\vec{O}$ Thoracic and Cardiovascular Surgery, 47, 577.

Feldman, P. S. and Kyriakos, M. (1972). Pulmonary $\vec{\omega}$ resection for metastatic sarcoma. Journal of Thoracic and Cardiovascular Surgery, 64, 784.

Hering, N., Templeton, J. Y., Haupt, G. J., and $\mathrm{O}$ Theodos, P. A. (1962). Primary sarcoma of the or lung. Diseases of the Chest, 42, 315.

Hovorka, J. (1966). Primary sarcomas of the lung. $\vec{\sigma}$ Casopis Lekaru Ceskych, 105, 561.

le Roux, B. T. (1968). Bronchial Carcinoma, pp. 136-141. E. and S. Livingstone, Edinburgh and $O$ London.

Lowell, L. M. and Tuhy, J. E. (1949). Primary음 chondrosarcoma of the lung. Journal of Thoracic $\stackrel{\Phi}{-}$ Surgery, 18, 476.

Mallory, T. B. (1936). Case 22441. Case records of the or Massachusetts General Hospital. New England. Journal of Medicine, 215, 837.

Montgomery, G. L. (1965). Textbook of Pathology, vol. I, p. 109. E. and S. Livingstone, Edinburgh and London.

Noehren, T. H. and McKee, F. W. (1954). Sarcoma $\stackrel{2}{\triangleright}$ of the lung. Diseases of the Chest, 25, 663.

Paterson, R. (1963). The Treatment of Malignant Disease by Radiotherapy, 2nd edition, pp. 3-5 and 412-413. Williams and Wilkins, Baltimore, and Arnold, London.

Rothe, G. (1965). Resections in primary sarcoma of the lungs. Zentralblatt für Chirurgie, 90, 883.

Schamaun, M. and Kunz, J. (1973). Clinic, therapyx and prognosis of primary sarcoma of the lung. Thoraxchirurgie, 21, 424.

Spencer, H. (1968). Pathology of the Lung, 2nd edition, pp. 908-914. Pergamon Press, Oxford.

Stout, A. P. (1948). Fibrosarcoma, the malignanto tumor of fibroblasts. Cancer, 1, 30.

Sutton, J. P., Parker, E. F., and Pratt-Thomas, H. R. (1968). Nonepithelial malignant tumors of the lung and mediastinum. American Surgeon, 34, N 605 .

Womack, N. A. and Graham, E. A. (1938). Mixed tumors of the lung, so-called bronchial or pul-w monary adenoma. Archives of Pathology, 26, 165.

Requests for reprints to: Mr. E. W. J. Cameron, $\mathscr{D}$ 10 Manse Street, Aberdour, Fife, Scotland. 